\title{
Successful Treatment of Sudden Bilateral \\ Case \\ Report Sensorineural Hearing Loss due to Atherosclerotic Vertebral Artery Occlusion: A Case Report
}

Takaaki Yamazaki, Hiroshi Moriwaki, Yasuyuki Tatsuta, Takehiko Sasaki, Mitsunori Shimazaki, and Mikio Nishiya

Objective: We report a case of sudden bilateral sensorineural hearing loss caused by atherosclerotic occlusion of vertebral artery which improved after angioplasty with stenting.

Case Presentation: The patient was a 71-year-old male. He was referred to our hospital from an otolaryngologist with severe, rapidly progressing hearing loss and vertigo. Cerebral angiography revealed occlusion of the predominant left vertebral artery, suggesting ischemia in the bilateral anterior inferior cerebellar artery (AICA) territories. Urgent balloon angioplasty was performed but after additional angioplasty, acute vessel reocclusion occurred associated with intimal dissection. Rescue stenting was performed and led to recanalization. After treatment, his hearing disturbance rapidly improved. Conclusion: Cochlear disorder caused by ischemia in the bilateral AICA regions may induce severe acute bilateral hearing loss. Acute revascularization is useful, but it is necessary to prepare stents for angioplasty and carefully evaluate whether treatment is indicated.

Keywords > sudden hearing loss, anterior inferior cerebellar artery, angioplasty and stenting, intracranial atherosclerosis, internal auditory artery

\section{Introduction}

Acute sensorineural hearing impairment with vertigo is related to vestibular dysfunction, such as Meniere's disease, vestibular neuronitis, and internal otitis, in many cases. However, ischemia in the anterior inferior cerebellar artery (AICA) territory may cause vertigo or hearing impairment. ${ }^{1)}$ In this study, we report a patient in whom ischemia in the bilateral AICA territories associated with atherosclerotic occlusion of the vertebral artery induced vertigo and bilateral sensorineural hearing loss with rapid

$\overline{\text { Department of Neurosurgery, Hakodate Neurosurgical Hospital, }}$ Hakodate, Hokkaido, Japan

Received: January 25, 2018; Accepted: April 21, 2018

Corresponding author: Takaaki Yamazaki. Department of Neurosurgery, Hakodate Neurosurgical Hospital, 1-4-12 Kamiyama, Hakodate, Hokkaido 041-8609, Japan

Email: takataka@mail.hnh.or.jp

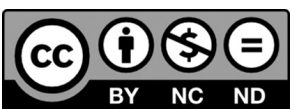

This work is licensed under a Creative Commons Attribution-NonCommercialNoDerivatives International License.

(C)2018 The Japanese Society for Neuroendovascular Therapy progression, and acute revascularization improved the symptoms.

\section{Case Presentation}

Case: A 71-year-old male.

Complaints: Bilateral hearing impairment, vertigo, and gait disorder.

Present illness: During the daytime, vertigo was noted while working. At night, it became exacerbated, and he consulted an emergency hospital. Under a tentative diagnosis of aural vertigo, he was referred to the Department of Otorhinolaryngology. An oral agent was administered, and follow-up was performed. However, the following day, bilateral hearing impairment appeared and rapidly progressed. In addition, gait disorder was noted, and he consulted the Department of Otorhinolaryngology of another hospital. Brain MRI revealed cerebral infarction, and he was referred to our hospital.

Medical history: Hypertension and diabetes mellitus. Detailed examination of the head indicated stenosis of the left vertebral artery 5 months prior to presentation. Clopidogrel at $75 \mathrm{mg}$ /day was administered. 


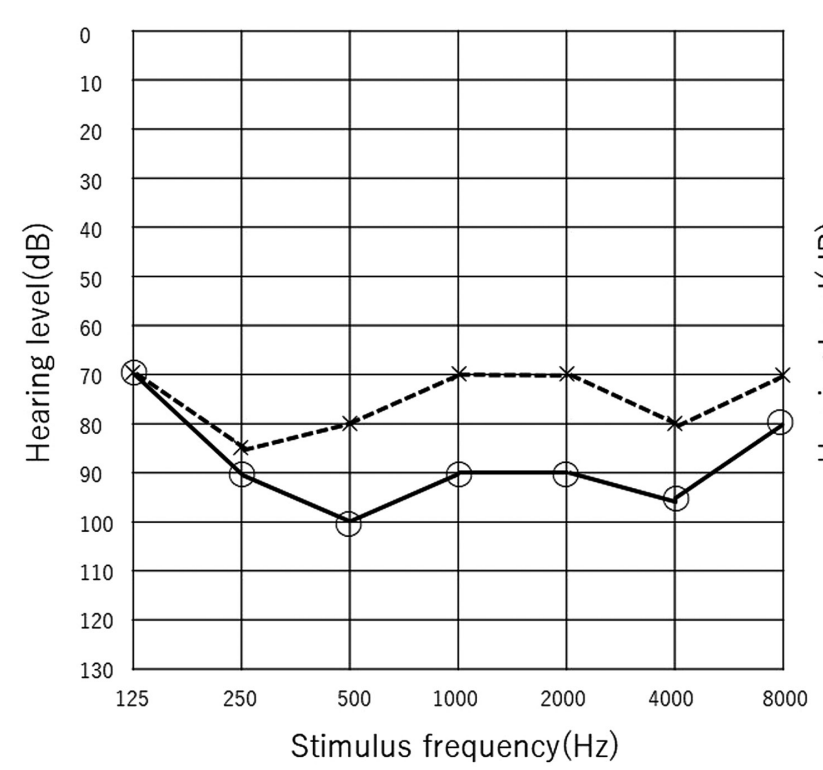

Air conduction Right ear: $\bigcirc$ Left ear: $\times \quad A$

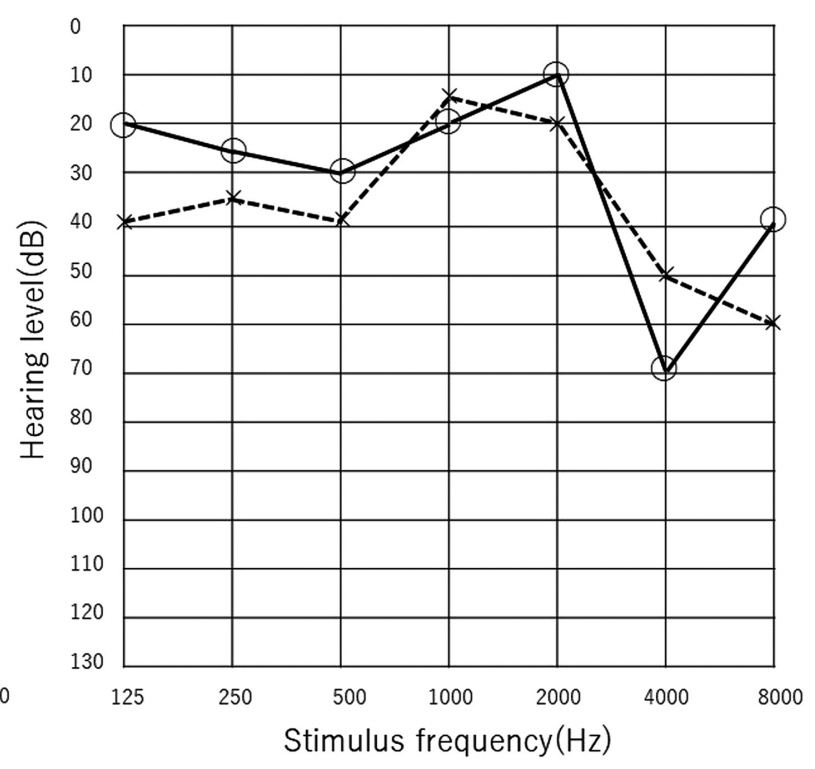

Air conduction Right ear: $\bigcirc$ Left ear: $\times \quad B$

Fig. 1 (A) An initial PTA demonstrated marked hearing loss in the bilateral ear. (B) Follow-up PTA indicated recovery of hearing. PTA: pure-tone audiogram

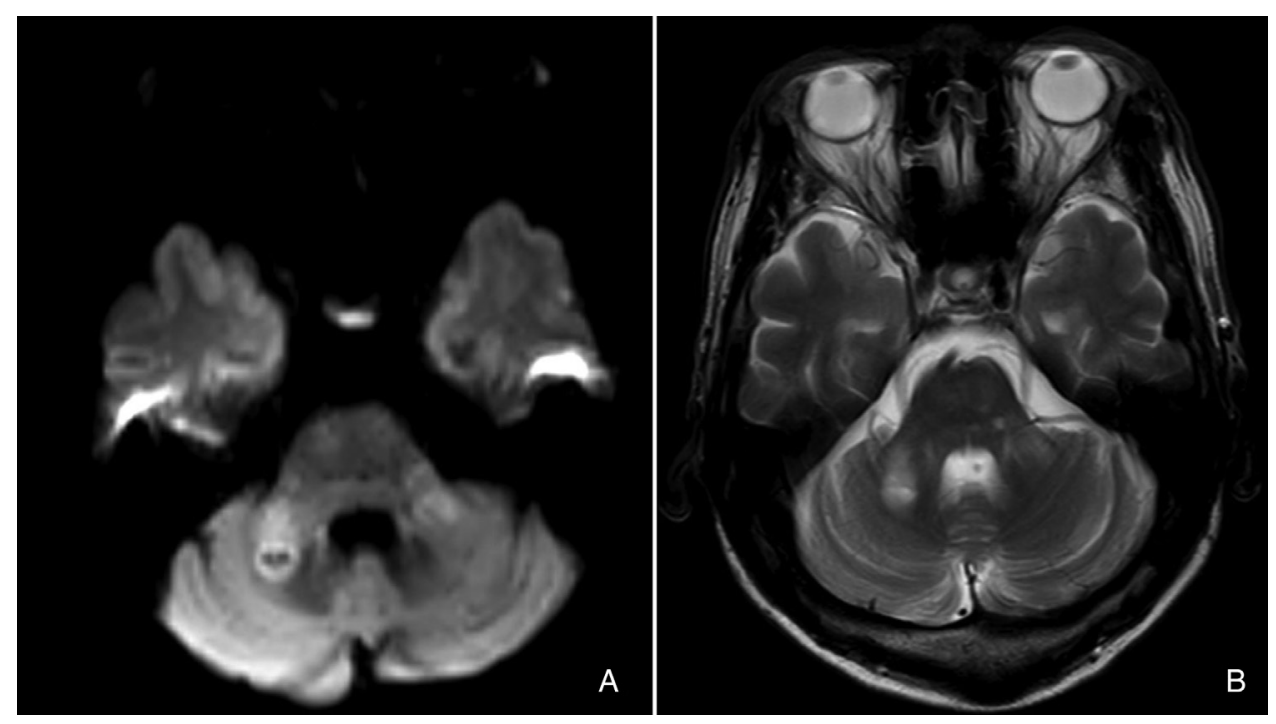

Fig. 2 MRI findings. DWI (A) and T2-weighted image (B) of the brain revealed hyper-dense lesions on the bilateral middle cerebellar peduncle. DWI: diffusion-weighted image

Family history: Not contributory.

Lifestyle: Smoking (20 cigarettes/day, 45 years).

Physical examination on admission: The Japan Coma Scale (JCS) score was 1 (severe bilateral hearing impairment required communication by writing). There was no facial sensory, movement, or ocular movement disorder. Horizontal rotatory nystagmus to the left and atactic gait was observed, and the results of left/right finger to nose tests were poor.

Pure-tone audiogram (PTA): Severe hearing impairment was noted (right: $92.5 \mathrm{~dB}$, left: $72.5 \mathrm{~dB}$ [4-minute method]) (Fig. 1A).

\section{Neuroradiological findings}

On brain MRI, high-signal intensity in the bilateral middle cerebellar peduncles was noted on diffusion-weighted images (DWIs) (Fig. 2A). A similar finding was also observed on T2-weighted images (Fig. 2B). Brain MRA 5 months before onset revealed stenosis of the left vertebral artery (Fig. 3A), and occlusion of the left vertebral artery and reduction in the visualization of the basilar artery were noted on MRA on admission (Fig. 3B). Cerebral angiography revealed occlusion of the predominantside left vertebral artery (Fig. 4A and 4B) and hypoplasia 


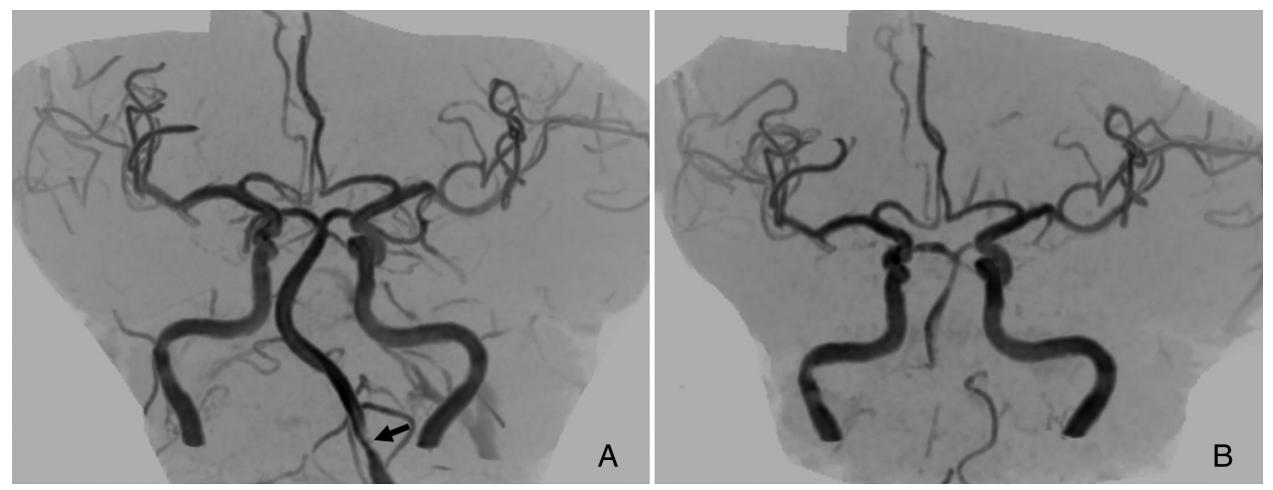

Fig. 3 (A) MRA performed on 5 months prior to presentation showed stenosis at the left vertebral artery (allow). (B) Occlusion of the left vertebral artery was noted on the same series on admission.
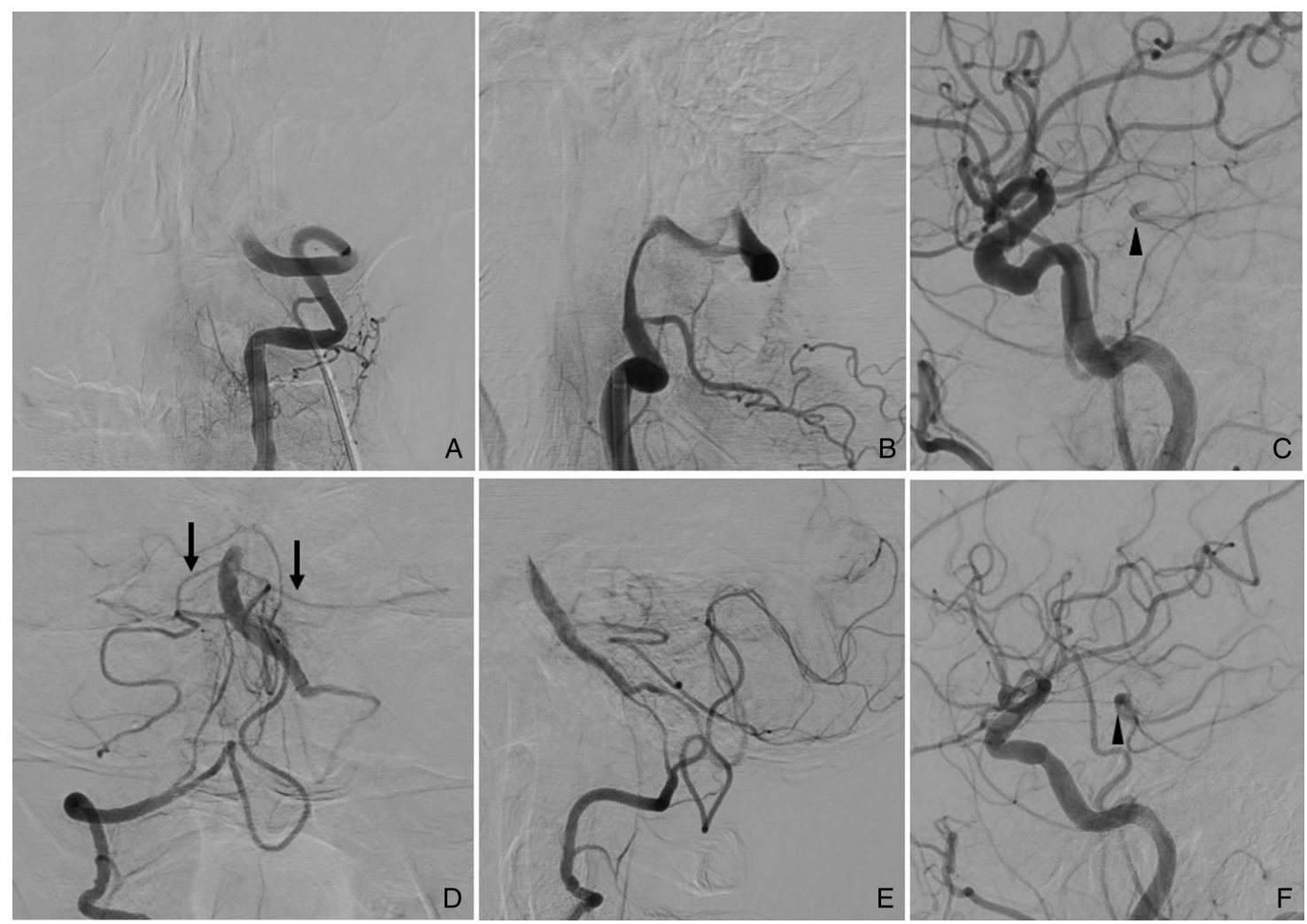

Fig. 4 (A) anteroposterior, (B) lateral left vertebral angiography demonstrated occlusion in the V4 segment. (D) anteroposterior, (E) lateral right vertebral angiogram. Antegrade injection into the proximal basilar artery, bilateral AICAs (arrows), and left-side posterior inferior cerebellar artery from the hypoplastic right vertebral artery. Left (C) and right (F) lateral carotid angiograms revealed poor retrograde injection into the basilar artery (arrowheads) through posterior communicating arteries. AICA: anterior inferior cerebellar artery

of the right vertebral artery peripheral to the posterior inferior cerebellar artery; the proximal basilar artery, contralateral left posterior inferior cerebellar artery, and bilateral anterior inferior cerebellar arteries were visualized (Fig. 4D and 4E). On bilateral common carotid angiography, the hypoplastic posterior communicating artery to basilar artery end was visualized (Fig. 4C and 4F). Based on these findings, occlusion of an atherosclerotic lesion of the left vertebral artery may have caused the marked ischemia in the bilateral AICA territories. As hearing had rapidly progressed, endovascular treatment was performed.

\section{Endovascular treatment}

Prior to treatment, two antiplatelet agents, clopidogrel at $300 \mathrm{mg}$ and aspirin at $200 \mathrm{mg}$ (loading dose), were administered. Systemic heparinization was performed to maintain 

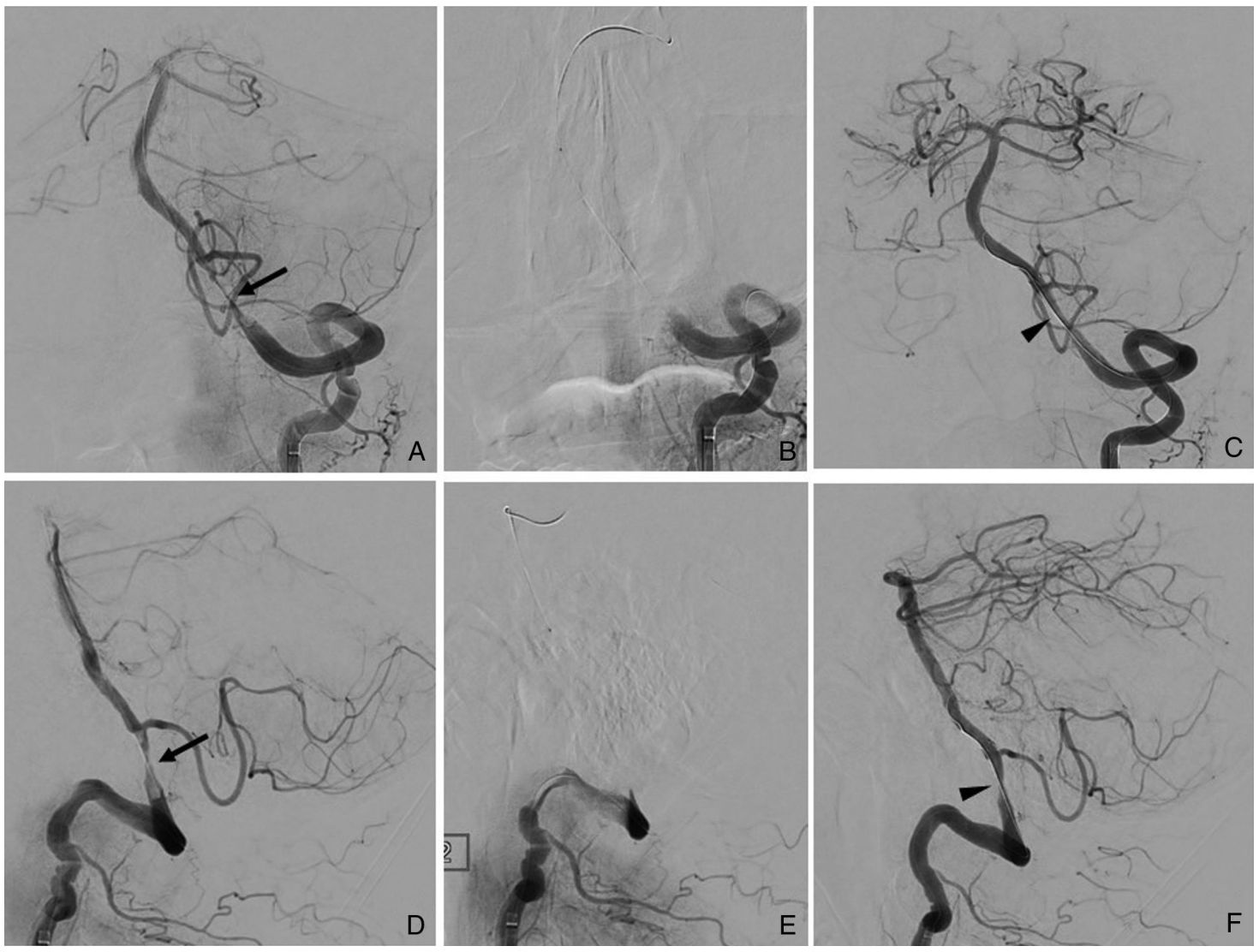

Fig. 5 A transverse wire was navigated to the left posterior cerebral artery through a microcatheter and used for angioplasties. After the first angioplasty with a 1.5-mm Gateway balloon (Stryker, Kalamazoo, MI, USA), reopening of the vertebral artery with significant residual stenosis (arrows) was observed from the left vertebral angiogram anteroposterior view (A) and lateral view (D). Additional balloon angioplasty with a 2-mm Gateway balloon was performed to improve the stenosis, but it was found to be acute occlusion of the vertebral artery (B) anteroposterior (E) lateral. Post-angioplasty and stenting, final vertebral angiography (C) anteroposterior (F) lateral demonstrating improved antegrade flow and moderate residual stenosis (arrowheads).

the activated clotting time (ACT) at $\geq 250$. Under local anesthesia, a 6 Fr FUBUKI guiding catheter (Asahi Intecc Co., Ltd., Aichi, Japan) was inserted into the left vertebral artery through the right femoral artery. A $200-\mathrm{cm}$ 0.014-inch CHIKAI microguidewire (Asahi Intecc Co., Ltd.) with a J-shaped tip was guided to the site of occlusion together with an Excelsior SL-10 microcatheter (Boston Scientific, Natick, MA, USA). At the site of occlusion, the microguidewire was inserted into the occluded portion and advanced up to the distal of the lesion. The microcatheter was then navigated over the wire across the occluded segment. Microcatheter angiography was performed to confirm the presence of the microcatheter in the true lumen, the microguidewire was advanced into the left posterior cerebral artery, and prolonged using an ASAHI EXTENSION NV extension wire (Asahi Intecc Co., Ltd.) to replace the microcatheter with a balloon catheter. A Gateway balloon for percutaneous angioplasty $1.5 \mathrm{~mm}$ in diameter and
$9 \mathrm{~mm}$ in length (Stryker, Kalamazoo, MI, USA) was introduced over the microguidewire, positioned to the occluded lesion and inflated to a nominal pressure of 6 atm through slow inflation ( $1 \mathrm{~atm} / 20$ seconds). Recanalization was obtained, but there was still significant residual stenosis (Fig. 5A and 5D). So the balloon was changed to a Gateway balloon $2.0 \mathrm{~mm}$ in diameter and $15 \mathrm{~mm}$ in length. This balloon was guided to the stenotic site, and inflation was performed, as described above, leading to acute reocclusion (Fig. 5B and 5E). Under a diagnosis of acute occlusion associated with intimal dissection, a Wingspan $2.5 \mathrm{~mm}$ in diameter and $15 \mathrm{~mm}$ in length (Stryker) was deployed/ placed across the lesion as rescue treatment. Although there was still moderate degree of residual stenosis, circulation was dramatically improved. The absence of changes was confirmed by repeat angiography (Fig. 5C and $\mathbf{5 F}$ ) after waiting for 15 minutes with the microwire in place, and the procedure was completed. 

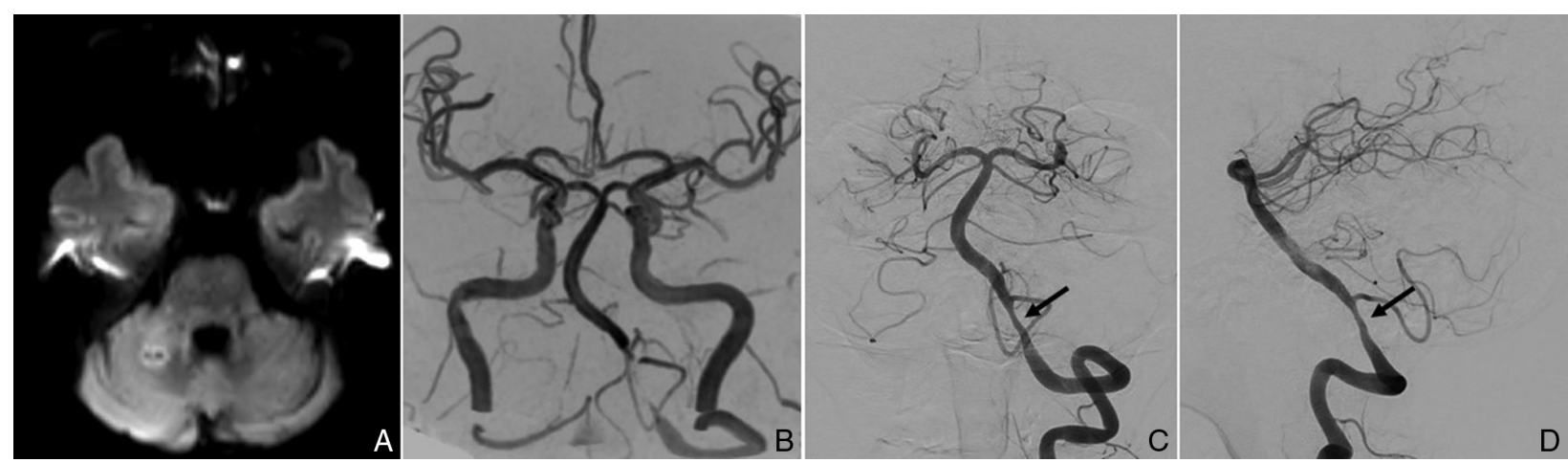

Fig. $6 \mathrm{MRI}$ and MRA performed on the day following treatment. There were no new lesions on DWI of the brain (A) and sufficient flow on angiography (B). Follow-up cerebral angiogram performed 12 months later (C) anteroposterior, (D) lateral revealed improvement in the degree of stenosis (arrows). DWI: diffusion-weighted image

\section{Course after treatment}

MRI (DWI) (Fig. 6A) the day after treatment did not reveal any new lesions. Furthermore, MRA confirmed favorable basilar artery blood flow (Fig. 6B). After treatment, his hearing rapidly improved. PTAs (Fig. 1B) indicated improvement of hearing (right: $20 \mathrm{~dB}$ [4-minute method], left: $22.5 \mathrm{~dB}$ [4-minute method]), and atactic gait gradually improved. Dual-antiplatelet therapy with clopidogrel at $75 \mathrm{mg} /$ day and aspirin at $100 \mathrm{mg} /$ day was continued. In addition to the medical management of hypertension and diabetes mellitus, lifestyle management, such as smoking cessation, was initiated. There has been no recurrence. Angiography 12 months after treatment did not demonstrate restenosis (Fig. 6C and 6D).

\section{Discussion}

Acute sensorineural hearing loss is considered to be an otorhinolaryngological symptom related to idiopathic hearing impairment or Meniere's disease. Many patients often consult the Department of Otorhinolaryngology and receive treatment. However, a similar symptom is sometimes induced by ischemic cerebrovascular disease involving the vertebrobasilar artery territory. The incidence of hearing impairment related to cerebral infarction involving the vertebrobasilar artery system ranges from $1.4 \%$ to $8 \%{ }^{1,2)}$ Although it differs among studies, this disorder is not so rare. According to one previous study, infarction involving the AICA region accounted for 5.5\% of cerebral infarction involving the vertebrobasilar artery system, but it accounted for $83.3 \%$ of vertebrobasilarartery-system cerebral infarction with acute hearing loss, and posterior inferior cerebellar artery infarction accounted for $11.9 \%$. In particular, ischemia in the AICA territories considered to be a primary etiological factor for hearing impairment.

The AICA originates from the $1 / 3$ caudal-side area of the basilar artery, and sometimes from the 1/3 middle area or the confluence of the bilateral vertebral arteries. It courses through the lateral part of the pons, crosses near the facial and vestibulocochlear nerve at the cerebellopontine angle, and bifurcates into the lateral and medial branches. At this segment, the internal auditory artery arises from the lateral branch. ${ }^{4)}$ The internal auditory artery, which supply the cochlea, branches from the meatal loop of the AICA, but rarely from the posterior inferior cerebellar or basilar arteries. ${ }^{3)}$ The internal auditory artery consists of one to several terminal arteries with little collateral circulations. Ischemia here may cause irreversible cochlear disorder.5) On the other hand, the cochlear nerve has an abundant collateral circulation, differing from the cochlea, and may resist ischemia. ${ }^{6}$ Lee et al. found that sudden hearing impairment was an important sign of AICA infarction, and concluded that hearing impairment and vertigo were caused by ischemic cochlear disorder in many patients. ${ }^{7)}$

In the present case, thrombotic occlusion of the predominant-side left vertebral artery led to hypoplastic distal right vertebral artery-mediated blood flow involving the proximal basilar artery, contralateral left posterior inferior cerebellar artery, and bilateral anterior inferior cerebellar arteries. In addition, a retrograde collateral pathway mediated by the posterior communicating artery was not sufficient, resulting in hypo-perfusion in the bilateral AICA territories. As a result, insufficient blood flow of the internal auditory artery as a terminal artery may have caused the cochlear dysfunction, leading to bilateral hearing loss.

Lee et al. reported that hearing recovered to some degree in approximately $80 \%$ of patients with hearing impairment 
related to cerebral infarction involving the vertebrobasilar artery region, whereas it was unfavorable in severe-status patients. ${ }^{1}$ One previous Japanese study examined hearing on initial consultation and its improvement rating in 46 patients with AICA syndrome related to stenosis/occlusion of the AICA, and reported that there were no hearing recovery in $16(44.4 \%)$ of 36 patients in whom it was possible to evaluate the improvement rating of hearing. Excluding 10 in whom it was unclear, hearing recovery was not favorable. ${ }^{8)}$ In addition, an experiment using guinea pigs demonstrated that hair cells were particularly damaged due to the effects of internal auditory artery occlusion on the cochlea; internal hair cells were damaged 3 hours after occlusion, and external hair cells 4 hours after occlusion. The disappearance of organum spirale hair cells was observed 6 hours after occlusion. ${ }^{9)}$ Therefore, it may be necessary to improve blood flow as early as possible in patients with severe hearing impairment.

Currently, when performing endovascular treatments for acute ischemic stroke due to intracranial large artery occlusion, mechanical thrombectomy with a stent retriever has been recommended as the first-line treatment. However, intracranial atherosclerotic stenosis (ICAS) is involved in the pathogenesis in some patients. Yoon et al. reported that ICAS was present in $22.9 \%$ of patients with acute cerebral ischemia who underwent endovascular treatment, and indicated the usefulness and safety of mechanical thrombectomy with a stent retriever as first-line treatment, followed by angioplasty with or without stenting for residual stenotic lesions. ${ }^{10)}$ In the present case, the presence of an atherosclerotic stenotic lesion on the left vertebral artery had been confirmed on previous MRA, and balloon angioplasty was primary performed. For angioplasty with a balloon, slow inflation is essential, but dissection may occur in some cases; therefore, a backup stent is necessary. In Japan, the self-expandable stent Wingspan is the only type approved for ICAS. However, this device is not always available. Thus, at our hospital, stents for stent-assisted coil embolization and balloon-mounted coronary stents are always prepared through approval by the Ethics Review Board to establish a backup system although these stents have not been approved for ICAS. In Japan, it is recommended that a Wingspan stent be indicated for vascular dissection during balloon angioplasty, acute/impending occlusion as a rescue treatment option, or additional treatment after angioplasty in cases in which there is no other effective treatment method. ${ }^{11)}$ In the present case, vascular dissection-related acute occlusion occurred after balloon angioplasty, requiring the use of a Wingspan stent for rescue stenting. Furthermore, ICAS-associated hemodynamic cerebral ischemia is considered to be a better indication for intracranial stents in comparison with branch atheromatous disease or thromboembolic occlusion. ${ }^{12)}$ In the present case, the recovery from hemodynamic cerebral ischemia may also have led to the rapid improvement in hearing. On the other hand, atherosclerotic debris being displaced or "snow-plowed" may occur during angioplasty or stenting, inducing occlusion of perforating arteries originating from an area adjacent to a stenotic lesion, ${ }^{13)}$ as indicated by the SAMMPRIS study. ${ }^{14)}$ In addition, the dura around the penetrating area of the V4 segment of the vertebral artery is much thicker than in other areas, and it forms a fibrous dural band; ${ }^{15)}$ therefore, stent deformity associated with head-movement-related mechanical stimuli and subsequent intimal injury-related restenosis/occlusion may occur. As such, endovascular treatment should be performed only when revascularization is considered to be more effective than best medical treatment.

\section{Conclusion}

We describe a case of sudden bilateral sensorineural hearing loss caused by atherosclerotic occlusion of vertebral artery successfully treated by angioplasty with stenting. Insufficient blood flow of the internal auditory artery as a terminal artery may have caused the cochlear dysfunction, leading to bilateral hearing loss. For angioplasty, it is necessary to prepare stents such as a Wingspan stent. Furthermore, it is important to carefully evaluate whether treatment is indicated.

\section{Disclosure Statement}

There is no conflict of interest regarding this article.

\section{References}

1) Lee H, Baloh RW: Sudden deafness in vertebrobasilar ischemia: clinical features, vascular topographical patterns and long-term outcome. J Neurol Sci 2005; 228: 99-104.

2) Huang $M H$, Huang CC, Ryu SJ, et al: Sudden bilateral hearing impairment in vertebrobasilar occlusive disease. Stroke 1993; 24: 132-137.

3) Lee H: Sudden deafness related to posterior circulation infarction in the territory of the nonanterior inferior cerebellar artery: frequency, origin, and vascular topographical pattern. Eur Neurol 2008; 59: 302-306. 
4) Naidich TP, Kricheff II, George AE, et al: The normal anterior inferior cerebellar artery. Anatomic-radiographic correlation with emphasis on the lateral projection. Radiology 1976; 119: 355-373.

5) Perlman HB, Kimura R, Fernandez C: Experiments on temporary obstruction of the internal auditory artery. Laryngoscope 1959; 69: 591-613.

6) Mazzoni A: Internal auditory canal arterial relations at the porus acusticus. Ann Otol Rhinol Laryngol 1969; 78: 797-814.

7) Lee H, Sohn SI, Jung DK, et al: Sudden deafness and anterior inferior cerebellar artery infarction. Stroke 2002; 33: 2807-2812.

8) Kitamura K, Otsuka K, Araki S, et al: Anterior inferior cerebellar artery (AICA) syndrome: a case report. OtoRhino-Laryngology, Tokyo; 2004; 47: 432-437.

9) Kimura R, Perlman HB: Arterial obstruction of the labyrinth. I. Cochlear changes. Ann Otol Rhinol Laryngol 1958; 67: 5-24.

10) Yoon W, Kim SK, Park MS, et al: Endovascular treatment and the outcomes of atherosclerotic intracranial stenosis in patients with hyperacute stroke. Neurosurgery 2015; 76: 680-686; discussion 686.

11) The Japan Stroke Society, The Japan Neurosurgical Society, The Japanese Society for Neuroendovascular Therapy. [Guidelines for the proper use of Wingspan stent system for intracranial atherosclerotic disease]. Jpn J Stroke 2014; 36: 151-162. (in Japanese)

12) Bang OY: Intracranial atherosclerosis: current understanding and perspectives. $J$ Stroke 2014; 16: 27-35.

13) Fiorella D, Derdeyn CP, Lynn MJ, et al: Detailed analysis of periprocedural strokes in patients undergoing intracranial stenting in stenting and aggressive medical management for preventing recurrent stroke in intracranial stenosis (SAMMPRIS). Stroke 2012; 43: 2682-2688.

14) Chimowitz MI, Lynn MJ, Derdeyn CP, et al: Stenting versus aggressive medical therapy for intracranial arterial stenosis. N Engl J Med 2011; 365: 993-1003.

15) Fine $\mathrm{AD}$, Cardoso A, Rhoton AL: Microsurgical anatomy of the extracranial-extradural origin of the posterior inferior cerebellar artery. J Neurosurg 1999; 91: 645-652. 